\title{
Optical properties of flavin mononucleotide: A QM/MM study of protein environment effects
}

\author{
Elena Cannuccia ${ }^{\mathrm{a}, \mathrm{b}, \mathrm{c}, *}$, Olivia Pulci $^{\mathrm{a}}$, Rodolfo Del Sole ${ }^{\mathrm{a}}$, Michele Cascella ${ }^{\mathrm{d}}$ \\ a ETSF, Dipartimento di Fisica, Università di Roma "Tor Vergata", via della Ricerca Scientifica 1, I-00133 Roma, Italy

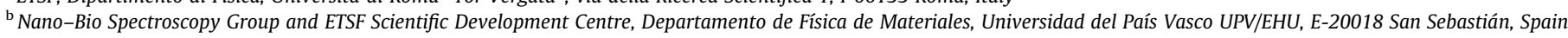 \\ ' IKERBASQUE, Basque Foundation for Science, E-48011 Bilbao, Spain \\ ${ }^{\mathrm{d}}$ Departement für Chemie und Biochemie, Universität Bern, Freiestrasse 3, 3012 Bern, Switzerland
}

\section{A R T I C L E I N F O}

\section{Article history:}

Received 18 May 2011

In final form 14 July 2011

Available online 26 August 2011

\section{Keywords:}

Flavin mononucleotide

QM/MM

Optical absorption

TDDFT

\begin{abstract}
A B S T R A C T
We investigate the optical properties of flavin mononucleotide (FMN) as chromophore of a bacterial protein. FMN is studied both in the oxidized and anionic state, by combining hybrid quantum mechanics/ molecular mechanics (QM/MM) dynamics with time-dependent density functional theory (TDDFT). Our first-principles calculations show that the inclusion of the protein biological environment is crucial to reproduce the experimental optical spectra.
\end{abstract}

(ㄷ) 2011 Elsevier B.V. All rights reserved.

\section{Introduction}

The recent development of ultrafast spectroscopy techniques has allowed to experimentally characterize the dynamics of chemical reactions in real time [1]. These methodologies have been of valuable significance not only to study simple systems in controlled environment, but also to address investigation of complex systems as, for example, liquid embedded proteins [2].

In this context, the photoactive groups hosted in biological macromolecules, play a crucial role. They work, in fact, as probes and are used to investigate local surroundings and monitor the systems dynamic. These optical probes can span from covalent moieties, within the same protein structure, to non-covalently bound cofactors, like transition metals or small aromatic molecules. The local environment significantly influences the optical response of such probes, thus, making them extremely useful to depict the dynamic changes occurring in their surroundings.

For a correct interpretation of the experimental data, reliable theoretical models, able to reconstruct the origin of any modulation of the experimental signal, are required. In particular, structural and dynamical properties of complex biomolecules can be addressed with sufficiently high level of accuracy by a hybrid combination of quantum mechanical and classical-molecular mechanics methods (QM/MM) [3].

\footnotetext{
* Corresponding author at: ETSF, Dipartimento di Fisica, Università di Roma "Tor Vergata", via della Ricerca Scientifica 1, I-00133 Roma, Italy.

E-mail address: elena.cannuccia@gmail.com (E. Cannuccia).
}

Similarly the excited state properties can be successfully described through the promising framework represented by timedependent density functional theory (TDDFT) [4]. Despite the use of more sophisticated methods may be sometimes advisable $[5,6]$, TDDFT represents the best compromise between the wished accuracy and the intrinsic complexity of systems of interest. TDDFT, in fact, allows to study systems involving chromophores as large as several tens of atoms, sampled over multiple configurations at finite temperature.

Within such framework, it has been recently possible to characterize the influence of the local environment over the redox properties of azurin from Pseudomonas aeruginosa [7], as well as its optical features [8]. Similar approaches have successfully determined the optical response of the chromophore in bacterial blue-protein [9] and in the green-fluorescent protein $[10,11]$. As a consequence, the theoretical investigation of optical probes is becoming a very active research field.

In this work, we present a characterization of the electronic structure and the optical spectra of oxidized and fully reduced FMN, bound to the flavodoxin from Clostridium beijerinckii bacterium (see Fig. 1) [12].

FMN and its derivatives are ubiquitous molecules which take part in biological reactions as coenzymes and photoreceptors of several proteins $[13,14]$ conferring them the role of tagging tools in bio-science.

The protein-cofactor interactions modulate the oxidationreduction potentials of FMN. As a result, FMN has a higher redox potential in the protein than in water. Moreover, when bound to 


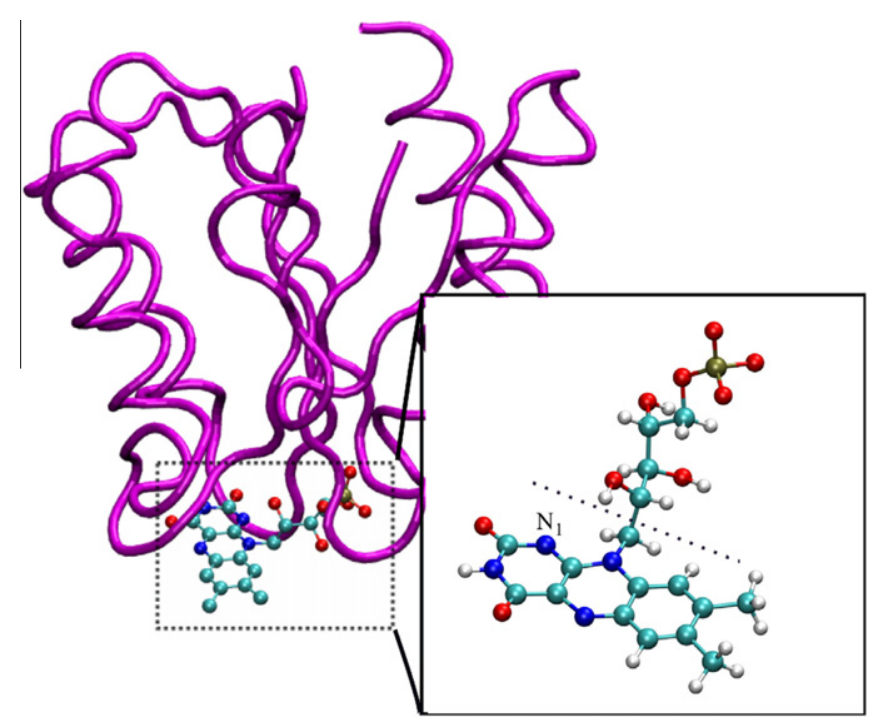

Fig. 1. Cartoon representation of the secondary structure of the flavodoxin. The FMN active site is zoomed and represented in balls and sticks. The QM/MM boundary, which crosses a covalent bond, is indicated by the dashed line.

the protein, FMN can be isolated in its radical semiquinone intermediate oxidation state, and its reduced state is anionic, unlike the neutral form present in solution. It is evident that the environment exerts a direct effect on the cofactor and also dictates the role played by flavins as electron-transfer in many physiological reactions. Moreover the environment influences the optical properties, which differ from those of FMN in solutions and/or in vacuum through the long range electrostatic potential and the structural modulation due to mechanical constraints.

Recently the time resolved electron transfer reaction in flavins was investigated by femtosecond spectroscopy [15]. For a full interpretation of experimental data, a thorough characterization of the absorption spectra of flavins in their different oxidation states is crucial. In fact, the sizable spectral differences between the various flavins redox states allow to monitor the events occurring in catalysis using the flavin optical response as a reporter [16]. This fact motivated our study of the different oxidation states in flavins and flavoproteins, that are at the origin of their different colors.

The paper is organized as follows: in Section 2 we summarize the details about the QM/MM computational setup and the TDDFT response calculations. The results concerning the optical properties of oxidized and fully reduced FMN as cofactors of flavodoxin are presented in Section 3. Conclusions and opened issues are summarized in Section 4.

\section{Computational details}

We considered the protein solvated by 8013 water molecules in a $67 \times 65 \times 61 \AA^{3}$ orthorhombic cell with periodic boundary conditions. Given the size of the simulation cell two periodically repeated proteins are never closer than $25 \AA$. As the isoalloxazine triple ring (see Fig. 1) is the active part in the light absorption, it has been treated at the DFT level [17]. The rest of the FMN, the protein and the solvent, were classically treated with the Amber force field (parm98) [18,19]. The QM/MM boundary, which crosses a covalent bond, has been treated using the well-known Hydrogencapping procedure [20]. The interaction between the classical and the quantum parts was described via a fully-Hamiltonian hierarchical coupling scheme [21].

The simulation was divided in three steps. First, the system was relaxed starting from the crystal structure [22]. Second, the classical Hamiltonian was substituted by the QM/MM one and the energy of the system was minimized. Third the system was thermalized at $300 \mathrm{~K}$, and different snapshots were sampled from its dynamics (details of the calculations in Ref. [23]). Such configurations were used to average the optical absorption spectra, calculated by TDDFT [17]. The effect of the protein environment has been taken into account by the QM/MM scheme.

\section{Results}

\subsection{Oxidized FMN}

As described in the previous section we performed a QM/MM dynamics simulation at $300 \mathrm{~K}$. For each configuration vertical transitions from the electronic ground state have been calculated.

The FMN isoalloxazine ring is an aromatic moiety characterized by strong covalent bonds, therefore, atoms do not undergo large fluctuations from their average positions within the sampled configurations. The position of the bands, at about 2.7 and $3.3 \mathrm{eV}$, is only slightly affected by the thermal fluctuations of the structure, while the intensity of the peak at $3.3 \mathrm{eV}$ can be modulated to $50 \%$, as the inset of Fig. 2 reports.

The conformation-averaged absorption spectrum is reported in Fig. 2, which is in very good qualitative agreement with the experiment [22]. A relative red shift of about $0.1 \mathrm{eV}$ is the major deviation from the experimental data plus a different relative intensity between the first and the second absorption peak. The spectrum shows two intense absorption regions with main transitions located in the blue-violet region, while the absorption in the red region is negligible. This explains the typical blue color which FMN confers to its solutions.

The two absorption bands have been characterized via an analysis of the Kohn-Sham orbitals involved in the optical transitions. The lowest energy absorption band is built up by transitions occurring from the highest occupied molecular $\pi$ orbital (HOMO), to the lowest unoccupied molecular $\pi^{*}$ (LUMO) orbital. The highest energy absorption band instead is originated by transitions from HOMO-2 to LUMO orbital. The above cited Kohn-Sham wavefunctions are shown in Fig. 3 together with the main transitions. The Kohn-Sham analysis reveals that the optical excitations involved have a reduced charge transfer character. This represents a basic condition to guarantee the good performance of the local exchange-correlation functionals used in the TDDFT simulations.

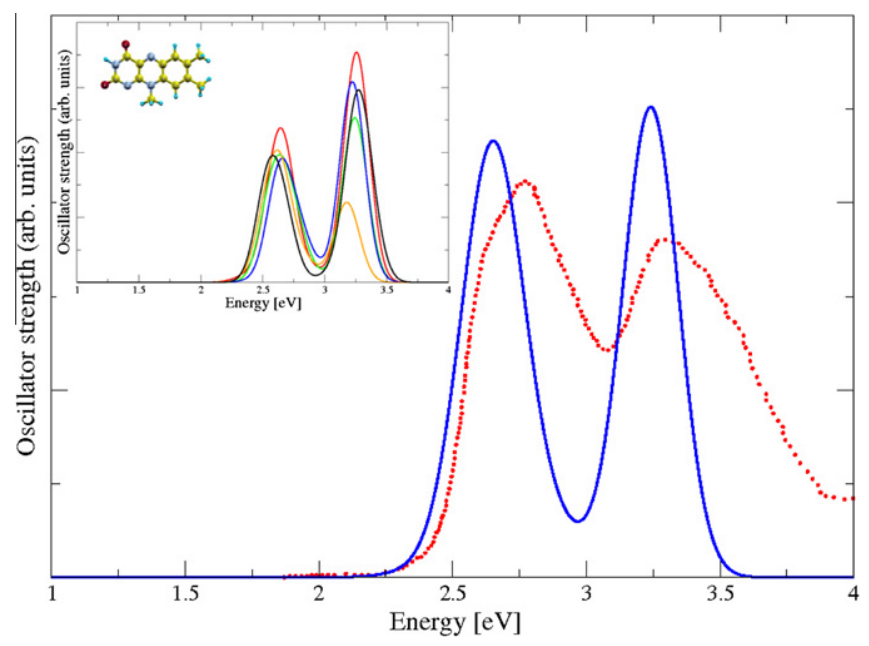

Fig. 2. TDDFT optical absorption spectra of oxidized FMN in flavodoxin. The (blue) solid line is the average computed spectrum. The (red) dotted line is the experimental curve, taken from Ref. [22]. In the inset the optical absorption spectra calculated on configurations sampled from a QM/MM simulation at $300 \mathrm{~K}$. (For interpretation of the references to color in this figure legend, the reader is referred to the web version of this article.) 
In order to shed more light on the coupling mechanism between the protein electrostatic environment and the electronic structure of the oxidized FMN, we repeated the spectra calculation nullifying the electrostatic field of the protein. The nearest environment of FMN, in fact, is characterized by negatively charged amino acids chains which generate a non homogeneous electrostatic field that can affect the electronic structure of FMN. The upper panel of Fig. 4 reports the computed absorption spectrum in the absence of the protein electrostatic potential. In these conditions, the bands appear to be shifted towards higher energies: $0.1 \mathrm{eV}$ for the first absorption band and about $0.2 \mathrm{eV}$ for the second one which is, at the same time, reduced in intensity.

The Kohn-Sham analysis reveals that the character of the energy transitions is also affected. Transitions from HOMO- 1 to LUMO build up the first absorption band, while the second one is given by transitions from HOMO-4 to LUMO Kohn-Sham wavefunctions. They involve, in general, deeper states with respect to the electrostatic potential switched-on case.

The protein scaffold influences the optical properties of FMN also by restraining its geometry in a distorted conformation. When bound to the protein, the isoalloxazine ring geometry results to be bent both in the X-ray structure [22] and in our QM/MM geometries, with the central aromatic ring being not perfectly planar. On the contrary, unconstrained geometry optimization in vacuum predicts a perfectly planar structure. Deformation of the perfectly planar structure of the flavin aromatic moiety affects the energies of the $\pi$ and $\pi^{*}$ orbitals, which are delocalized over the whole structure, and therefore, strongly influences the optical response of the system. The lower panel of Fig. 4 reports a comparison of the in vacuum optical spectra of FMN in both the protein-restrained and unrestrained optimized geometries [24]. In the unrestrained geometry both the absorption bands are strongly shifted towards the red by $0.25-0.3 \mathrm{eV}$. We verified that the Tamm-Dancoff approximation (TDA) does not affect the blue shift of the spectrum. We attributed therefore it to the mechanical effects of the protein rather than an artifact of the TDA

\subsection{Reduced anionic FMN}

The reduced form of FMN in water is neutral, while it is anionic when bound a protein. The anionic form is stabilized by a strong hydrogen-bond between the $N_{1}$ atom (see Fig. 1 ) and the backbone of the protein.

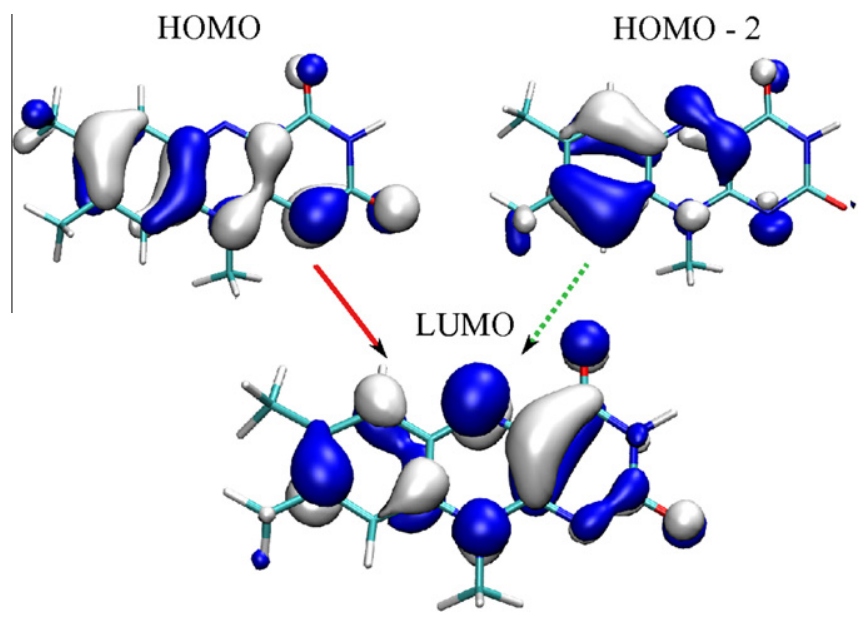

Fig. 3. The Kohn-Sham wavefunctions which dominate the optical excitations. The (red) solid arrow highlights the transitions building up the lower energy absorption peak, while the higher one is shown by the (green) dashed arrow. (For interpretation of the references to color in this figure legend, the reader is referred to the web version of this article.
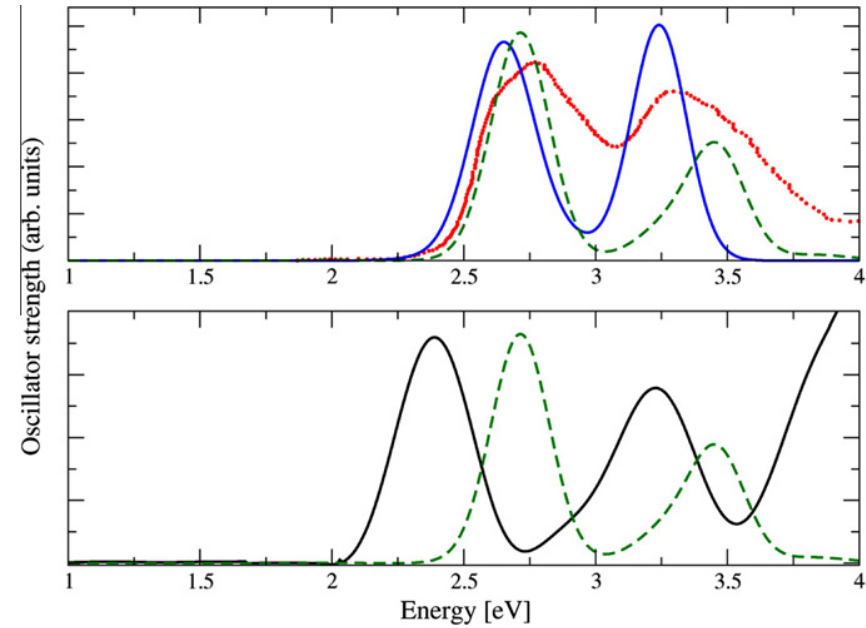

Fig. 4. Optical absorption spectra of oxidized FMN in flavodoxin. The TDDFT spectrum with [(blue) solid line] and without [(green) dashed line] the effect of the electrostatic potential is shown (upper panel) together with the experiment [22] [(red) dotted line]. In the lower panel the optical absorption spectra of oxidized FMN in flavodoxin obtained with [(green) dashed line] and without [(black) solid line] the mechanical constraint of the protein environment. The second case corresponds to consider FMN in vacuum and to use a real space-real time [25] approach to calculate the optical absorption spectrum. (For interpretation of the references to color in this figure legend, the reader is referred to the web version of this article.)

The optical absorption spectrum of the reduced form of FMN was analyzed by following a similar procedure as for the oxidized form. Particular care was put in the equilibration of the system, as the excess of charge in the QM region could produce electron-spill out artifacts that would affect the quality of the dynamics. Fig. 5 reports the result from our calculations, in comparison with the experiment and the in vacuum optical spectrum. We find a qualitative agreement in the position of the first band, despite this time the shape of the line seems less good. The Kohn-Sham analysis reveals that the absorption band is dominated by $\pi-\pi^{*}$ transitions from HOMO and HOMO-1 to LUMO orbitals.

Contrary to the oxidized neutral form, the reduced anionic FMN results to be rather sensitive to the local geometrical fluctuations of the environment (see inset of Fig. 5). Given the presence of strongly $\mathrm{H}$-bonded moieties, as well as $\pi$-stacked aromatic groups

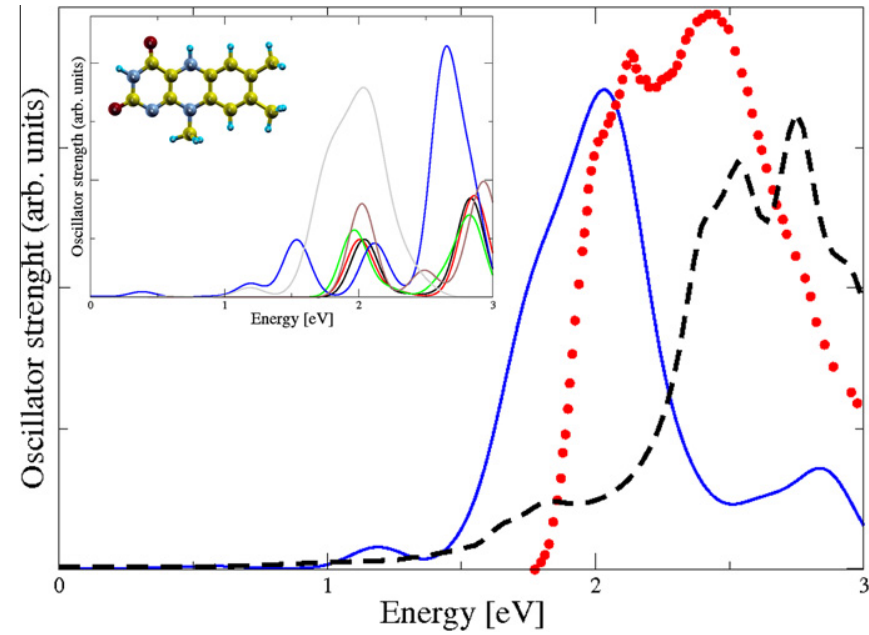

Fig. 5. Optical absorption spectra of reduced anionic FMN in flavodoxin. The averaged TDDFT spectrum over seven snapshots and the experiment are shown in (blue) solid line and (red) dots respectively. The in vacuum spectrum is shown in (black) dashed line. (For interpretation of the references to color in this figure legend, the reader is referred to the web version of this article.) 
like Trp90, the discrepancy between our calculation and the experimental absorption line may be due to the presence of charge transfer states involving FMN to the protein surrounding [15]. We stress here that our QM/MM protocol excludes the possibility of determining such states, because the protein environment is strictly treated at the classical level of theory, and that anyway intermolecular charge-transfer states would be poorly described by TDDFT with local functionals.

Moreover in our simulation the charge is delocalized over the chromophore because the QM/MM setup confines the additional charge of FMN in the simulation box. In natural condition, indeed, the charge would diffuse through the surrounding protein affecting the dynamics of the whole system and causing therefore a possible discrepancy in the spectrum.

\section{Conclusions}

QM/MM simulations coupled to TDDFT calculations were used to describe the optical properties of FMN bound to a complex environment such as flavodoxin from $C$. beijerinckii.

The protein acts on the optical properties of oxidized FMN both through its electrostatic field and by inducing structural deformations on the ligand. The electrostatic field of the protein produces a slight shift of the absorption bands toward the red; on the contrary, the disruption of coplanarity of the aromatic moiety induces a strong blue shift of the bands. Reasonable agreement with the experiment was found also for the reduced anionic form of FMN. In this case, the optical response of the system is more sensitive to local geometrical fluctuations. Discrepancies with the experiment may be due to the presence of charge-transfer states, which cannot be reliably described within the level of approximation chosen in our study, and the confinement of the additional charge due to the computational setup.

In conclusion our study underlines the importance of the environment effects on the optical properties of FMN. Using QM/MM combined with TDDFT we were able to shed light on different optical spectra of FMN in the two redox states, that are at the base of its use as optical marker.

\section{Acknowledgments}

We acknowledge funding by the European Community through e-I3 ETSF project (Contract Number 211956), the IRSES project SIMTECH (No. 246937), and by the Swiss National Science Foundation (Grant No. PP02_118930). Computing power was provided by CINECA, CASPUR and ENEA CRESCO supercomputing centres. One of the authors (EC) acknowledges Prof. Ursula Röthlisberger for fruitful discussions.

\section{References}

[1] A.H. Zewail, Femtochemistry: Ultrafast dynamics of the chemical bond, World Scientific Publishing, 1994.

[2] M. Sugisaki, M. Fujiwara, D. Kosumi, R. Fujii, M. Nango, R.J. Cogdell, H. Hashimoto, Phys. Rev. B 81 (24) (2010) 245112, doi:10.1103/PhysRevB.81. 245112.
[3] A. Warshel, M. Levitt, J. Mol. Biol. 103 (1976) 227.

[4] G. Onida, L. Reining, A. Rubio, Rev. Mod. Phys. 74 (2) (2002) 601.

[5] Y. Ma, M. Rohlfing, C. Molteni, Phys. Rev. B 80 (24) (2009) 241405, doi:10.1103/ PhysRevB.80.241405.

[6] A. Mosca Conte et al., J. Chem. Theory Comput. 5 (7) (2009) 1822.

[7] M. Cascella, A. Magistrato, I. Tavernelli, P. Carloni, U. Röthlisberger, Proc. Natl. Acad. Sci. 103 (52) (2006) 19641, doi:10.1073/pnas.0607890103.

[8] M. Cascella, M.A. Cuendet, I. Tavernelli, U. Röthlisberger, J. Chem. Phys. B 111 (34) (2007) 10248, doi:10.1021/jp071938i.

[9] X. Lopez et al., J. Am. Chem. Soc. 127 (35) (2005) 12329.

[10] T. Laino, R. Nifosì, V. Tozzini, Chem. Phys. 298 (1-3) (2004) 17, doi:10.1016 j.chemphys.2003.10.040

[11] M.A.L. Marques, X. López, D. Varsano, A. Castro, A. Rubio, Phys. Rev. Lett. 90 (25) (2003) 258101.

[12] S. Wilkinson, M. Young, J. Bacteriol. 177 (1995) 439

[13] A.M.E. Eduardo Silva, RCS Pub., 2006.

[14] K. Yagi, T. Yamano, Japan Scientific Societies Press: Tokyo and University Park Press: Baltimore, 1980.

[15] N. Mataga et al., J. Chem. Phys. B 106 (2002) 8917.

[16] T. Drepper, R. Huber, A. Heck, F. Circolone, A. Hillmer, J. Büchs, K. Jaeger, Appl. Environ. Microbiol. 76 (2010) 5990.

[17] All DFT and TDDFT calculations have been performed using the PBE XC functional [26], the core-valence interactions has been considered via dispersion corrected atom-centered pseudopotentials [27] and the electronic wavefunctions were expanded in plane waves with 70 Ry cutoff. Linearresponse TDDFT has been evaluated within the Tamm-Dancoff approximation [28]. All calculations have been carried out by the CPMD code [29] while for the optical response of FMN in vacuum, we opted for a real space-real time approach by using the octopus code $[25,30]$. In the latter case the wave functions are represented by their value over a set of points in real space. The separation between points, or spacing, was fixed on $0.18 \AA$ (which corresponds to a cut-off kinetic energy of $85 \mathrm{Ry})$. Spheres of radii $5 \AA$ and $8 \AA$, built around each atom was chosen as the simulation region for the oxidized and the anionic form, respectively. The wave functions were evolved for a period of $4 \times 10^{-16} \mathrm{~s}$

[18] T. Cheatham, P. Cieplak, P. Kollman, J. Biomol. Struct. Dyn. 16 (1999) 845.

[19] W.D. Cornell, P. Cieplak, C.I. Bayly, I.R. Gould, K.M. Merz, D.M. Ferguson, D.C Spellmeyer, T. Fox, J.W. Caldwell, P.A. Kollman, J. Am. Chem. Soc. 117 (1995) 5179.

[20] U. Röthlisberger, P. Carloni, Drug-target binding investigated by quantum mechanical/molecular mechanical (QM/MM) methods, in: M. Ferrario, G. Ciccotti, K. Binder (Eds.), Computer Simulations in Condensed Matter Systems: From Materials to Chemical Biology Volume 2, Lecture Notes in Physics, Vol. 704, Springer, Berlin/ Heidelberg, 2006, p. 449.

[21] A. Laio, J. VandeVondele, U. Röthlisberger, J. Chem. Phys. 116 (2002) 6941.

[22] M. Ludwig, K. Pattridge, A. Metzger, M. Dixon, M. Eren, Y. Feng, R. Swenson, The crystal structure is taken from the database <www.rcsb.org >: PDB code 5nll (1997).

[23] Structural relaxation was performed by means of $2 \mathrm{~ns}$ classical molecular dynamics (MD) starting from the crystal structure [22]. Subsequently the energy, with the $\mathrm{OM} / \mathrm{MM}$ Hamiltonian, was minimized by repeated cycles of simulated annealing. Finally the system was gradually thermalized at $300 \mathrm{~K}$ starting from an initial temperature of $50 \mathrm{~K}$ within 2 ps of MD and finally 5 snapshots every 500 fs have been sampled from its dynamics.

[24] E. Cannuccia, O. Pulci, M. Palummo, V. Garbuio, R. Del Sole, Phys. Stat. Sol. 5 (2008) 2542.

[25] A. Castro et al., Phys. Stat. Sol. B 243 (2006) 2465.

[26] J.P. Perdew, K. Burke, M. Ernzerhof, Phys. Rev. Lett. 80 (4) (1998) 891.

[27] I.-C. Lin, M.D. Coutinho-Neto, C. Felsenheimer, O.A. von Lilienfeld, I. Tavernelli, U. Röthlisberger, Phys. Rev. B 75 (2007) 205131.

[28] S. Hirata, M. Head-Gordon, Chem. Phys. Lett. 314 (3-4) (1999) 291.

[29] M. Eichinger, P. Tavan, J. Hutter, M. Parrinello, J. Chem. Phys. 110 (21) (1999) 10452.

[30] M.A.L. Marques, A. Castro, G.F. Bertsch, A. Rubio, Comput. Phys. Commun. 151 (1) (2003) 60. 